\title{
Resin bonded bridges: techniques for success
}

\author{
K. A. Durey, ${ }^{1}$ P. J. Nixon, ${ }^{2}$ S. Robinson ${ }^{3}$ and M. F. W.-Y. Chan ${ }^{4}$ \\ VERIFIABLE CPD PAPER
}
- Gain a contemporary understanding of the role of resin bonded bridges in replacing missing teeth.
- Learn how to improve survival and aesthetics of resin bonded bridges.
- A 'quick reference' summary of things to consider clinically and technically, to improve outcome.

Resin bonded bridges are a minimally invasive option for replacing missing teeth. Although they were first described over 30 years ago, evidence regarding their longevity remains limited and these restorations have developed an undeserved reputation for failure. This article provides a brief review of the literature regarding bridge success and continues to highlight aspects of case selection, bridge design and clinical procedure which may improve outcome.

\section{INTRODUCTION}

Resin bonded or resin retained bridges (RBBs/RRBs) are minimally invasive fixed prostheses which rely on composite resin cements for retention. These restorations were first described in the 1970s and since this time they have evolved significantly. The first type of RBB was the Rochette Bridge, which relied on the retention generated by resin cement tags through a characteristic perforated metal retainer. ${ }^{1}$ However, longevity of this type of restoration was limited and in an effort to address this, methods of altering the surface of the metal retainer to enhance micromechanical retention were developed. ${ }^{2}$ The term 'Maryland Bridge' resulted from the development of a type of electrochemical etching at the University of Maryland. More recently bridge retention has been enhanced by the development of resin cements which bond chemically to both the tooth surface and the metal alloy.

From a clinician's perspective, the main advantage of RBBs is that, in comparison to conventional bridge preparations, they are conservative of tooth structure. ${ }^{3}$

"Specialist Registrar in Restorative Dentistry, ${ }^{2,4}$ Consultant in Restorative Dentistry, Leeds Dental Institute Clarendon Way, Leeds, LS2 9PU; ${ }^{3}$ Senior Lecturer in Clinical Dentistry, University of Queensland, University of Queensland, Turbot Street, Brisbane, Australia ${ }^{*}$ Correspondence to: Dr Kathryn Durey Email:kathryndurey@hotmail.co.uk

\section{Refereed Paper}

Accepted 16 June 2011

DOI: $10.1038 /$ sj.bdj.2011.619

${ }^{\circ}$ British Dental Journal 2011; 211: 113-118
By using a RBB it is possible to provide a fixed replacement for missing teeth which is essentially reversible and does not compromise the abutment tooth. This is especially important for young patients who may be more likely to experience endodontic complications as a result of extensive tooth preparation.

Despite this recognised advantage, the role of RBBs as definitive restorations remains somewhat controversial due to a lack of long term prospective data regarding success. The majority of information is based on the results of longitudinal studies, many of which have been poorly controlled, used a variety of cements and preparation techniques making it difficult to isolate factors affecting outcome. ${ }^{4}$

Recent systematic reviews have estimated the five-year survival rates for bridgework as $87.7 \%$ for resin bonded prostheses $^{4}$ and just over $90 \%$ for conventional bridges depending on design. ${ }^{5}$ Although these rates are lower than the 94.5\% success $^{6}$ reported for implant retained single crowns over the same five year follow up, resin bonded bridgework has the advantages of being less invasive, requiring a shorter total treatment time and less financial commitment.

In contrast to these favourable estimations of RBB success, Hussey et al. ${ }^{7}$ reported high failure rates when they used the number of recement fees claimed to gauge the success of RBBs in NHS general practice. Additionally, a recent study of RBB designs employed by dentists in both general practice and hospital settings reported that a high proportion of practitioners used unfavourable techniques. ${ }^{8}$ It seems reasonable to assume that with improved education and careful planning, outcome could be improved.

The aim of this article is to re-evaluate the role of RBBs in fixed prosthodontics and provide a guide for practitioners with regard to case selection, bridge design and clinical techniques in order that successful outcomes may be achieved.

\section{FACTORS AFFECTING SUCCESS}

\section{Case selection}

\section{i) Patient factors}

Restoration of missing teeth aims to improve oral function, aesthetics and restore occlusal stability. However, intervention should be considered carefully as in some cases it may be detrimental to the remaining dentition. ${ }^{9-11}$

General factors such as the health, age of the patient, their expectations, local factors related to dental health and the missing tooth itself need to be taken into account. For example in older patients with reduced manual dexterity it may be appropriate to accept a shortened dental arch rather than replacing a lost posterior unit. If a tooth must be replaced, a RBB may be preferable to a removable partial denture (RPD) especially where there is a history of significant periodontal disease or dental caries. ${ }^{9}$ As they are minimally invasive, RBBs can also provide a temporary option for young 
patients who have suffered the early loss of an anterior tooth. This situation would otherwise condemn the patient to years of denture wear until growth has ceased and an implant or definitive bridge can be considered.

RBBs have the advantages of taking minimal clinical time ${ }^{12}$ and rarely requiring anaesthetic, therefore they may be appropriate for patients who are apprehensive of dental treatment or unable to commit to more involved treatment involving multiple appointments. However, the patient should still be dentally motivated and caries and periodontal disease should be under control before embarking on fixed prosthodontics. In addition, managing expectations with regard to aesthetic outcome and longevity should be considered an important part of treatment planning. ${ }^{13}$ If expectations are unrealistic, patient satisfaction with the final result is likely to be low.

\section{ii) Abutment tooth selection}

When selecting abutment teeth, investigations should be carried out to ensure endodontic and periodontal health. Periodontal support should be assessed considering bone levels and root configuration. Although a history of periodontal disease and reduced bone support does not exclude bridgework (Fig. 1), the use of abutments with active periodontal disease should be avoided as increased functional loading may increase the rate of periodontal destruction. ${ }^{14}$

Coronally, there should be sufficient enamel available for bonding. The dentitions of hypodontia patients are frequently associated with a degree of microdontia reducing the amount of tooth structure available. Surface area may also be compromised if teeth are restored or where
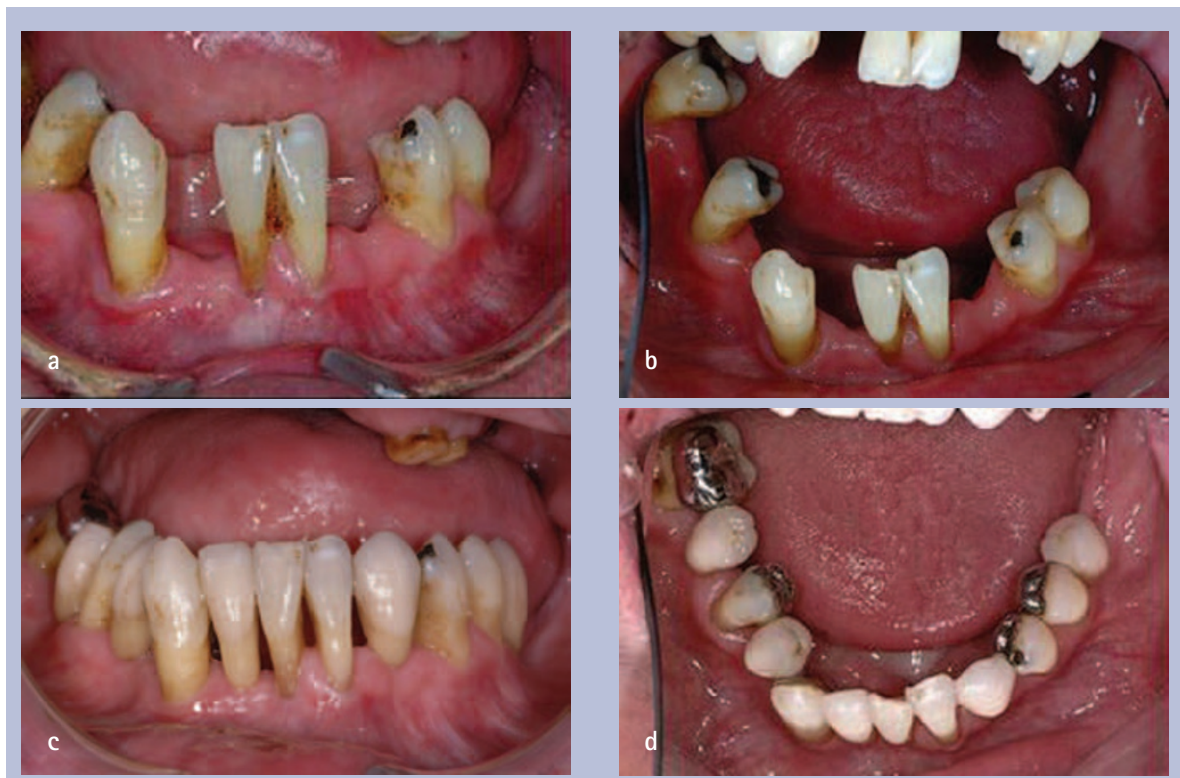

Fig. 1 a) Older patient with a history of successfully treated periodontal disease and dissatisfaction with partial denture. b) Remaining teeth lingually tilted with increased mobility. c) Provision of multiple (5) cantilever RBBs mimicking root exposure and staining of natural teeth. d) Note novel bucco-occlusal retaining wing used on lingually tilted molar tooth

there has been significant tooth wear. Additionally, the alignment or angulation of teeth may affect the degree to which a retainer can be extended. Crowding may reduce access and rotations may mean that full wraparound is difficult to achieve. An unconventional approach may be necessary, for example in Figure 1, where a buccal retaining wing has been used on a lingually tilted molar in an effort to avoid the undercut lingual area that proved difficult to access.

If periodontal support and coronal condition are favourable, any teeth, including retained deciduous teeth, ${ }^{15}$ can act as abutments over an appropriate span. Deciduous molars can make particularly good abutments as they are multirooted and have a large coronal surface area which allows full extension of the retainer wing. The roots of retained deciduous teeth are likely to have undergone some resorption and have reduced length however, they may also be ankylosed and so are well placed to act as abutments.

\section{iii) Occlusal features}

When planning for RBBs, a detailed assessment of both static and dynamic occlusal relationships is crucial to optimise success. A wax up on articulated casts gives a valuable view from the palatal aspect aiding the assessment of the amount of interocclusal space available for the retainer wings and pontics. ${ }^{13}$ It is important that the pontic is not involved in guidance during mandibular excursive movements. ${ }^{16}$ If this is unachievable, guidance should be shared with other natural teeth.

If there is insufficient space for an aesthetic pontic, adjustment of opposing teeth could be considered. Alternatively space
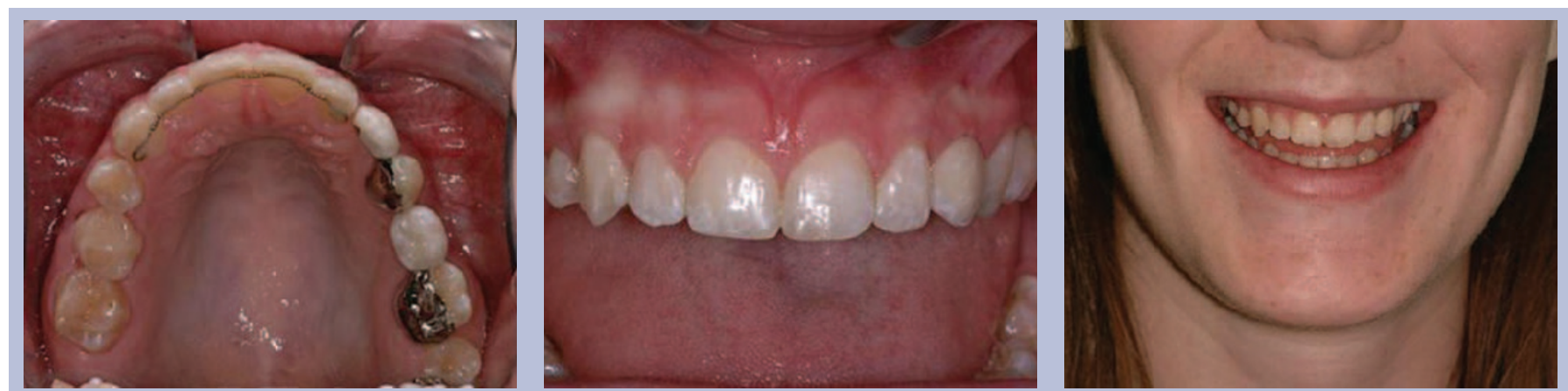

Fig. 2 Hypodontia case demonstrating two cantilever RBBs to replace UL 3 and ULE. Note the extent of coverage of metal retainers, characterisation of porcelain work and ovate style pontic to achieve good aesthetics 
may be gained with localised anterior composite build ups to adjust guidance patterns or by cementing the restoration at an increased OVD on the retainer. ${ }^{17}$ With both of these options the occlusion would then be allowed to re-establish over a period of months through passive eruption. ${ }^{18}$ Cementing restorations high does not appear to increase the risk of abutment teeth proclining or the restoration debonding, ${ }^{19}$ however, the authors suggest that this technique should be used to make only modest and controlled changes to the occlusion.

Parafunctional forces increase the likelihood of restoration failure, especially where the occlusion has not been accounted for. Any habits should be identified during the assessment phase and the patient should be counselled to avoid habits like nail and pen biting. Where bruxism is suspected the prescription of a night guard or occlusal splint should be considered.

\section{Bridge design}

It has been widely reported that RBBs are more successful as cantilevers than as fixed-fixed restorations. ${ }^{20-23}$ Despite this evidence a high number of dentists continue to use fixed-fixed designs and double abutments. ${ }^{8}$

Resin bonded bridges with multiple abutments are more likely to debond due to the differential movement of abutment teeth, especially where occlusal contact involves the natural tooth surface. In these cases occlusal force leads to the tooth and the retainer being driven apart causing failure of the cement lute. ${ }^{19}$ Where two abutment teeth have been used it is unlikely that both retainers will debond simultaneously. When only one retainer fails, the bridge is likely to remain in situ promoting the development of caries beneath the failed retainer. ${ }^{20,21,24}$

There are, however, some situations in which a fixed-fixed design may be the most appropriate. These include large pontic spans and where abutment teeth are small and sufficient surface area for retention can only be gained by using one abutment at either end of the span. It has also been suggested that fixed-fixed RBBs can provide a form of orthodontic retention, particularly where teeth have been de-rotated. ${ }^{25}$ However, it is the view of the authors that orthodontic retention should be maintained separately to restorative treatment, either with removable orthodontic retainers or orthodontic bonded wire retainers. If a fixed-fixed design is required, contact in excursive movements and intercuspation should be on the retainer only. ${ }^{19}$

\section{i) Retainer wing coverage}

The surface area covered by an RBB retainer has been shown to affect retention. It is accepted that $180^{\circ}$ wraparound retainers constitute the ideal design, but this must be balanced with the demand for aesthetics. Retainers on posterior teeth may be extended to include coverage of the palatal and lingual cusps and a proportion of the occlusal surface (Fig. 2) to increase the surface area and improve retention. If necessary, the surface area for bonding can be maximised by crown lengthening, either with conventional periodontal flaps or with electrosurgery (Fig. 3). Electrosurgery is particularly relevant for young patients who have short clinical crown heights, a substantial proportion of whom present following orthodontics wearing retainers which can be associated with gingival hyperplasia. If teeth are restored, fillings should be replaced with fresh composite restorations, which will bond more favourably to the resin cement enhancing retention of the bridge. ${ }^{26}$

\section{ii) Technical features}

Any flexing of the metal bridge retainer exerts stress on the cement lute that
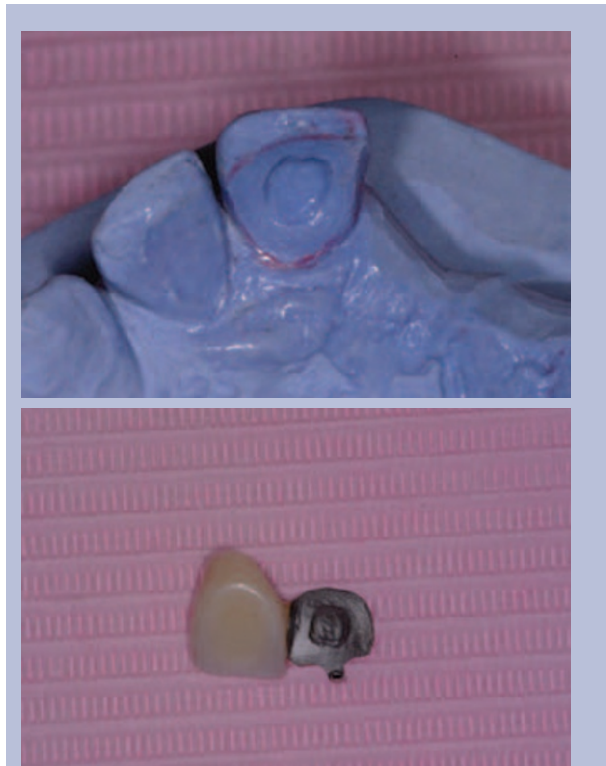

Fig. 4 Extension of retainer wing into existing palatal access cavity to improve resistance

and retention form. Also note incisal seating lug

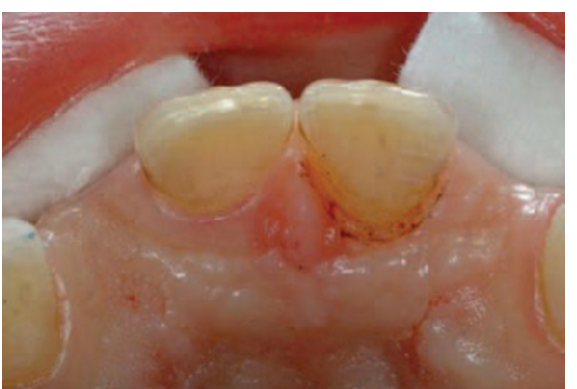

Fig. 3 Upper central incisor following lengthening of clinical crown height using electrosurgery

eventually leads to fatigue failure. ${ }^{27}$ Base metal alloys are highly rigid and therefore can be used in thin section without risk of flexing, making them ideally suited for use in RBB retainers. In vitro research has shown that base metal retainers of less than $0.7 \mathrm{~mm}$ thickness have less resistance to dislodgement ${ }^{28}$ and therefore $0.7 \mathrm{~mm}$ as a minimum dimension should be stipulated in the technical prescription. Where there is insufficient interocclusal space to accommodate a retainer of this thickness, teeth can be reduced to create space or the bridge can be cemented high as previously described. ${ }^{17,18}$ Clinicians should verify adequate thickness of the metal retainer before cementation to ensure sufficient rigidity, for example using an Iwansson crown gauge (UnoDent Ltd, Witham, Essex, UK).

A locating tag or seating lug should be extended over the incisal edge of anterior teeth (Fig. 4) to help to locate the retainer
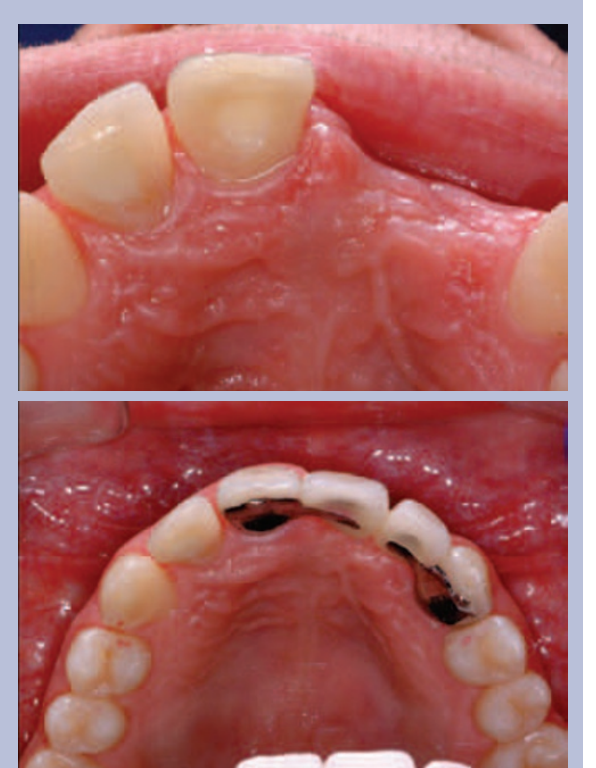
correctly and resist cervical displacement of the retainer during cementation. It can be removed with a bur after cementation and the metal polished as needed.

\section{iii) Aesthetics}

The aesthetics of a RBB are determined by the retainer wing, the porcelain work and how the soft tissues are managed. Metal connectors may shine-through translucent incisors causing them to appear grey and in fact Djemal et al. ${ }^{19}$ reported that the metal of the retainer was the most common reason for patient dissatisfaction with their RBB.

Greying can be reduced to a degree by the use of opaque cement and careful retainer design, avoiding extending the metal to within $2 \mathrm{~mm}$ of the incisal edge, where the enamel becomes relatively more translucent. In cases where the retainer cannot be disguised by opaque cements, it may be necessary to reconsider the choice of abutment tooth or place composite labially as a veneer.

The shade of the porcelain should be conveyed to the technician by means of a shade map, which can include details of characterisation features if appropriate (Fig. 2). The shade should be taken in natural light at the beginning of the appointment when the teeth are hydrated. A good quality digital photograph with the chosen shade tab in situ can be a valuable aid for the technician.

\section{iv) Pontic design}

Several alternatives for pontic design have been described based on the pontic-ridge relationship. The most commonly used of these is the modified ridge lap pontic, which allows reasonable aesthetics and facilitates hygiene. In aesthetically critical areas, the authors' preferred alternative to this is the ovate pontic, which has a convex profile to the soft tissue fitting surface helping to create a good emergence profile (Fig. 2). When designing the pontic, it is important to relate the gingival level to that of the adjacent natural teeth.

\section{Clinical techniques}

\section{i) Need for tooth preparation}

The need for tooth preparation for RBBs is a subject of debate. Previous research used more extensive preparations to enhance
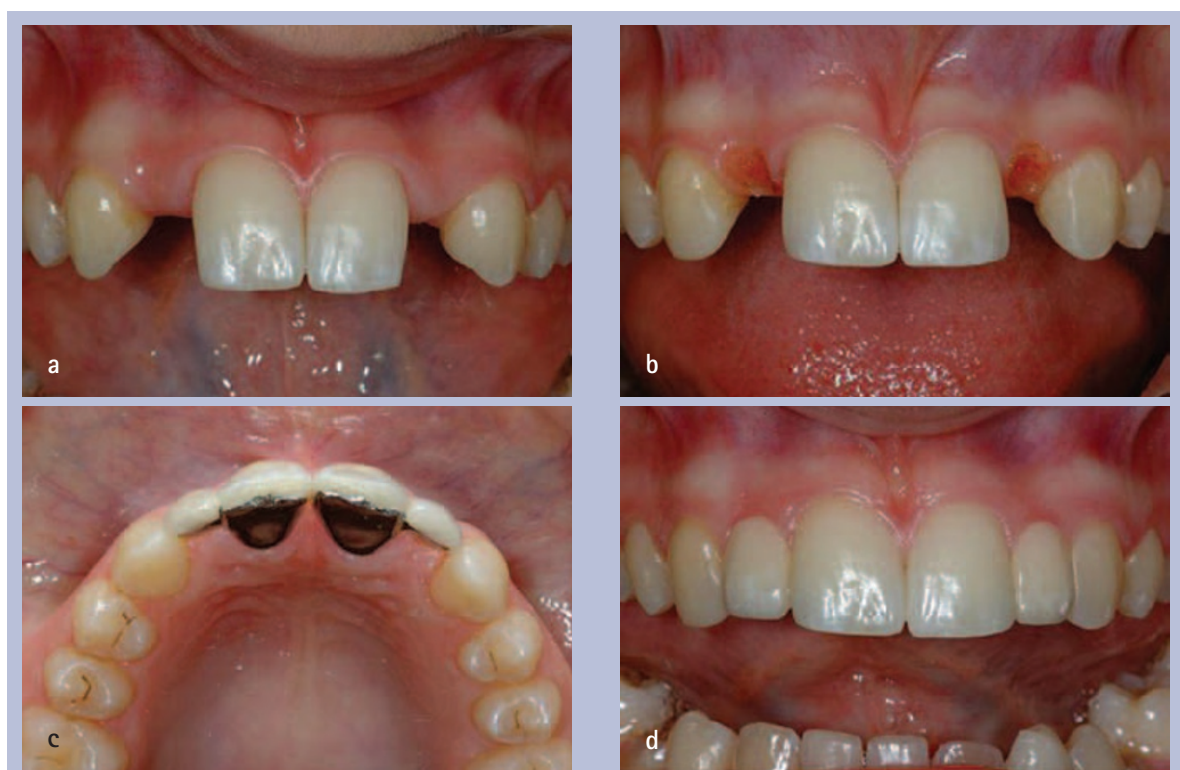

Fig. 5 a) Young patient presenting with developmentally missing lateral incisors. Note the central incisors are barrel shaped and the canines diminutive. b) Ridge preparation at the pontic site, note the central incisors and canines have been built up using composite resin to improve dimensions. c) Resin bonded bridges in situ replacing the lateral incisors. d) The emergence profile created following ridge preparation and use of an ovate pontic gives a pleasing aesthetic result

retention, ${ }^{29}$ however, most authorities now advocate minimal preparation, within enamel, ${ }^{30}$ or no preparation at all. ${ }^{17,19}$

Vertical grooves are the particular feature which has been identified as reducing stresses on the cement bond ${ }^{31}$ and increasing resistance to debonding forces. ${ }^{29,32}$ However, preparation involves irreversible damage to abutment teeth for what is reported to be only a limited benefit, ${ }^{19}$ and even when minimal preparation is intended, dentine exposure is likely during preparation. ${ }^{24}$ Bond strength to dentine is lower than that that can be achieved to enamel which may affect bridge retention. Additionally dentine exposure increases the chance of sensitivity between appointments and the risk of caries if the area is not sealed adequately at cementation.

A situation in which more extensive preparation can be justified is when teeth are restored. Preparation may be developed into restorations to produce longitudinal grooves, occlusal rests and boxes on posterior teeth, and into access cavity restoration on anterior teeth. This helps to promote axial loading and creates resistance form (Fig. 4).

\section{ii) Cementation}

Developments in resin cements have helped to increase restoration longevity. Early composite resin materials exhibited degradation and reduced bond strength with time. In contrast, Panavia (Karrary Co. Ltd, Osaka, Japan) demonstrates prolonged high bond strengths. This is due to formation of a chemical bond between the phosphate group of the cement monomer and the oxide layer of the metal retainer. Sandblasting to create micromechanical interlocking should be carried out before cementation to further enhance retention.

RBB cementation requires an uncontaminated, etched and primed enamel or dentine surface to generate maximum bond strengths. In vitro research has shown that achieving uniform and ideal etching of enamel surfaces is variable, especially on lingual surfaces of lower posterior teeth where moisture control is difficult. ${ }^{33}$ Audenino et $a .^{34}$ found that the use of rubber dam during cementation reduced the risk of the restoration debonding; however, in contrast, Marinello et al. ${ }^{35}$ reported the isolation method used had no significant effect on bridge outcome. It is the experience of the authors that, if patients are compliant, adequate moisture control can be achieved in the upper anterior region using the cotton wool rolls and saliva ejectors. Elsewhere in the mouth rubber dam is advisable and a split dam technique can be utilised to facilitate seating of the restoration. 


\section{iii) Ridge preparation}

A disadvantage of all bridgework is its inability to replace soft tissue. In cases with vertical ridge defects, pink porcelain $^{36}$ or composite may be used to replicate the gingival margins. However, the amount of soft tissue this can replace is limited as the restoration becomes bulky and compromises oral hygiene. Matching gingival shade and characterisation is also challenging.

When there is adequate ridge height, soft tissue management aims to create a realistic emergence profile and interdental papilla. This can be done clinically by defining the pontic site with a high speed bur or electrosurgery ${ }^{37}$ immediately before impression taking (Fig. 5). Alternatively, the master cast may be relieved in the lab and the soft tissues adjusted at fit. If the clinician is unable to alter the cast themselves, the depth of relief required (taking in to account the compressibility of the tissues clinically at the pontic site), should be conveyed to the technician.

Where electrosurgery has been carried out and the patient is wearing a RPD, this can be relined to help to maintain the gingival contour between appointments. If there is any relapse, electrosurgery can be repeated or the pontic modified at bridge fit.

\section{DEALING WITH FAILURE}

Biological reasons for failure include caries and periodontal disease but these occur relatively rarely. ${ }^{4}$ To prevent complications oral health education, encompassing oral hygiene instruction and advice regarding diet and the use of fluoride, should be provided at the treatment planning stage and finalised following bridge cementation. Where a fixed-fixed design has been used, patients should be warned of the risk of one retainer debonding and to report this immediately if they feel that the bridge is loose.

The most common technical reason for RBB failure is debonding. ${ }^{5}$ Although authors have reported that debonding does not appear to affect patient satisfaction ${ }^{19,38}$ and there is usually limited damage to abutment teeth, it is an inconvenience. Other technical problems which may necessitate remake of the bridge include structural damage and shade match deterioration which can be a result of natural tooth discoloration or porcelain changes.

\section{Table 1 Factors related to success of resin bonded bridges}

\section{Case selection}

- Patient selection: are they motivated/compliant?

- Does the space need to be restored? What options are there for restoration?

- Abutment tooth quality: is the tooth periapically and periodontally healthy? Is there periodontal support adequate? Is there sufficient enamel surface area for bonding and how translucent is the enamel?

- Tooth position: is spacing and alignment of natural teeth favourable? How large is the pontic span and will the abutment(s) support this span length?

- Occlusal assessment: is there sufficient space for a pontic of the right shape and size and the retainer, or does this need to be created?

- Parafunctional habits: are there any habits that can be eliminated or do they need to be managed as part of the treatment plan?

- Expectations: has enough information been provided? Are the patient's expectations realistic with regard to aesthetics and longevity?

Bridge design

- Retainer of $0.7 \mathrm{~mm}$ thickness

- Full retainer extension as allowed by aesthetic demands

- Minimal ICP contact

- Careful management of excursive contacts to avoid undue forces on pontic

- Use of an ovate pontic were aesthetics are important

Clinical techniques

- Replace existing restorations with composite

- Ensure adequate clinical crown height or crown lengthen to increase bonding area if necessary

- Create space for the restoration: opposing tooth adjustment, preparation of abutment tooth or cement at increased OVD

- Preparation: for unrestored teeth use minimal preparation, on restored teeth, extend preparations into restorations to increase resistance form

- Assess shade accounting for opaque cement and possible grey shine through of retainer wing

- Prepare the pontic site to improve gingival profile when needed for aesthetics

- Excellent moisture control during cementation and use of a resin cement with a phosphate monomer eg Panavia

- Protect the final result: provide a night guard or orthodontic retention if required

If a bridge debonds there are two options: remake or recement. If a one off event such as trauma has resulted in decementation, recementing the restoration may well be appropriate. However, studies have shown that once a bridge has debonded it is more likely to fail again ${ }^{39}$ and recementing for a second time is generally ill advised as replacing the bridge has been found to have a higher success rate. ${ }^{35,39}$ This is probably because in the majority of failed cases, there is an inherent problem with bridge design which may have been present at initial cementation and/or developed since. With this in mind, the restoration itself should be examined and the patient should be reassessed from an occlusal perspective: have they developed a new parafunctional habit or has the occlusion changed in ICP or lateral excursion as a result of restoration or tooth wear of adjacent or opposing teeth?

If the decision is made to recement a $\mathrm{RBB}$, the metal retainer should be air abraded and any cement residue removed carefully from the tooth before attempting this. Where the restoration is cantilevered, recementation is usually straightforward. Where there is a fixed-fixed design and only one side is loose, attempts can be made to remove the retainer that is still in place with the help of an ultrasonic scaler. Alternatively, depending on the length of span, the debonded retainer can 
be sectioned and the bridge left in situ as a cantilever. ${ }^{2}$

\section{CONCLUSION}

Resin bonded bridges can be highly effective in replacing missing teeth, restoring oral function and aesthetics and result in high levels of patient satisfaction. They represent a minimally invasive, cost effective and long lasting treatment modality. Given thorough patient assessment and the use of careful clinical techniques (Table 1), the authors suggest that RBBs should be considered more frequently as the restoration of choice for short spans.

The authors are very grateful for the excellent standard of technical support provided by Mr Kevin Wilson, Senior Dental Technician, Leeds Dental Institute

1. Howe D F, Denehy G E. Anterior fixed partial dentures utilizing the acid-etch technique and a cast metal framework. J Prosthet Dent 1977; 37: 28-31.

2. St George G, Hemmings K, Patel K. Resin-retained bridges re-visited. Part 1. History and indications. Prim Dent Care 2002; 9: 87-91.

3. Edelhoff D, Sorensen J A. Tooth structure removal associated with various preparation designs for anterior teeth. J Prosthet Dent 2002; 87: 503-509.

4. Pjetursson B E, Tan W C, Tan K, Bragger U, Zwahlen $M$, Lang N P. A systematic review of the survival and complication rates of resin-bonded bridges after an observation period of at least 5 years. Clin Oral Implants Res 2008; 19: 131-141.

5. Pjetursson B E, Bragger U, Lang N P, Zwahlen M. Comparison of survival and complication rates of tooth-supported fixed dental prostheses (FDPs) and implant-supported FDPs and single crowns (SCs). Clin Oral Implants Res 2007; 18 Suppl 3: 97-113.

6. Jung $R$ E, Pjetursson B E, Glauser $R$, Zembic $A$, Zwahlen $\mathrm{M}$, Lang N P. A systematic review of the 5 -year survival and complication rates of implantsupported single crowns. Clin Oral Implants Res 2008; 19: 119-130.

7. Hussey D L, Wilson $\mathrm{NH}$. The provision of resinbonded bridgework within the General Dental Services 1987-1997. Prim Dent Care 1999; 6: 21-24.

8. Patsiatzi $E_{1}$ Grey N J. An investigation of aspects of design of resin-bonded bridges in general dental practice and hospital services. Prim Dent Care 2004; 11: 87-89.

9. Jepson N J, Moynihan P J, Kelly P J, Watson G W, Thomason J M. Caries incidence following restoration of shortened lower dental arches in a randomized controlled trial. Br Dent J 2001; 191: 140-144.

10. Knoernschild K L, Campbell S D. Periodontal tissue responses after insertion of artificial crowns and fixed partial dentures. J Prosthet Dent 2000; 84: 492-498.

11. Rashid S A, Al-Wahadni A M, Hussey D L. The periodontal response to cantilevered resin-bonded bridgework. J Oral Rehabil 1999; 26: 912-917.

12. Verzijden C W, Creugers N H, van't Hof M A. Treatment times for posterior resin-bonded bridges. Community Dent Oral Epidemio/ 1990; 18: 304-308.

13. Tredwin C J, Setchell D J, George G S, Weisbloom M Resin-retained bridges as predictable and successful restorations. Alpha Omegan 2007; 100: 89-96.

14. Lindhe J, Svanberg G. Influence of trauma from occlusion on progression of experimental periodontitis in the beagle dog. J Clin Periodontol 1974 1:3-14.

15. Robinson S, Chan M F. New teeth from old: treatment options for retained primary teeth. Br Dent $J$ 2009; 207: 315-20.

16. Briggs $P$, Dunne $S$, Bishop K. The single unit, single retainer, cantilever resin-bonded bridge. Br Dent J 1996; 181: 373-379.

17. Ibbetson R. Clinical considerations for adhesive bridgework. Dent Update 2004; 31: 254-256, 258 260 passim.

18. Poyser N J, Porter R W, Briggs P F, Chana H S, Kelleher M G. The Dahl Concept: past, present and future. Br Dent J 2005; 198: 669-676.

19. Djemal S, Setchell D, King P, Wickens J. Long-term survival characteristics of 832 resin-retained bridges and splints provided in a post-graduate teaching hospital between 1978 and 1993. J Oral Rehabil 1999; 26: 302-320.

20. Chan A W, Barnes I E. A prospective study of cantilever resin-bonded bridges: an initial report. Aust Dent J 2000; 45: 31-36.

21. Olin PS, Hill E M, Donahue J L. Clinical evaluation of resin-bonded bridges: a retrospective study. Quintessence Int 1991; 22: 873-877.

22. van Dalen $A$, Feilzer $A J$, Kleverlaan C J. A literature review of two-unit cantilevered FPDs. Int J Prosthodont 2004; 17: 281-284

23. Kern M. Clinical long-term survival of two-retainer and single-retainer all-ceramic resin-bonded fixed partial dentures. Quintessence Int 2005; 36: 141-147.

24. Bassi G S, Youngson C C. An in vitro study of dentin exposure during resin-bonded fixed partial denture preparation. Quintessence Int 2004; 35: 541-548.
25. Garnett M J, Wassell R W, Jepson N J, Nohl F S Survival of resin-bonded bridgework provided for post-orthodontic hypodontia patients with missing maxillary lateral incisors. Br Dent J 2006; 201: 527-534.

26. Walls A W, Nohl F S, Wassell R W. Crowns and other extra-coronal restorations: resin-bonded metal restorations. Br Dent J 2002; 193: 135-135, 141-142.

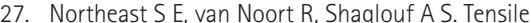
peel failure of resin-bonded $\mathrm{Ni} / \mathrm{Cr}$ beams: an experimental and finite element study. J Dent 1994; 22: 252-256.

28. Ibrahim A A, Byrne D, Hussey D L, Claffey N. Bond strengths of maxillary anterior base metal resinbonded retainers with different thicknesses. J Prosthet Dent 1997: 78: 281-285.

29. De Kanter R J, Creugers N H, Verzijden C W, Van't Hof M A. A five-year multipractice clinical study on posterior resin-bonded bridges. J Dent Res 1998; 77: 609-614.

30. Botelho M. Design principles for cantilevered resinbonded fixed partial dentures. Quintessence Int 2000; 31: 613-619.

31. Ziada H M, Orr J F, Benington I C. Photoelastic stress analysis in perforated (Rochette) resin bonded bridge design. J Oral Rehabil 2000; 27: 387-393.

32. Creugers N H, de Kanter R J, Verzijden C W, van't Hof M A. [Five year survival of posterior adhesive bridges. Influence of bonding systems and tooth preparation]. Ned Tijdschr Tandheelkd 1999; 106: $250-253$.

33. Hobson R S, Crotty T, Thomason J M, Jepson N J. A quantitative study of enamel acid etch patterns on surfaces used for retention of resin-bonded fixed prostheses. Eur J Prosthodont Restor Dent 2005; 13: $123-128$.

34. Audenino G, Giannella G, Morello G M, Ceccarelli M, Carossa S, Bassi F. Resin-bonded fixed partial dentures: ten-year follow-up. Int J Prosthodont 2006; 19: 22-23.

35. Marinello C P, Kerschbaum T, Heinenberg B et al. Experiences with resin-bonded bridges and splints a retrospective study. J Oral Rehabil 1987; 14: 251-260.

36. Alani A, Maglad A, Nohl F. The prosthetic management of gingival aesthetics. Br Dent J 2011; 210: $63-69$.

37. Elder A R, Djemal S. Electrosurgery: a technique for achieving aesthetic and retentive resin-bonded bridges. Dent Update 2008; 35: 371-374, 376.

38. Creugers N H, De Kanter R J. Patients' satisfaction in two long-term clinical studies on resin-bonded bridges. J Oral Rehabil 2000; 27: 602-607.

39. Creugers N H, Kayser A F. An analysis of multiple failures of resin-bonded bridges. J Dent 1992; 20: $348-351$. 